\title{
Direct Extension
}

National Cancer Institute

\section{Source}

National Cancer Institute. Direct Extension. NCI Thesaurus. Code C120271.

The spread or migration of cancer cells through the wall of an org an into surrounding organs and/or adjacent tissues. 